\title{
New observation strategies for the solar UV spectral irradiance
}

\author{
Gaël Cessateur ${ }^{1, *}$, Jean Lilensten ${ }^{2}$, Thierry Dudok de $\mathrm{Wit}^{3}$, Ali BenMoussa ${ }^{4}$, and Matthieu Kretzschmar ${ }^{3,4}$ \\ 1 Physical-Meteorological Observatory / World Radiation Center, Davos, Switzerland \\ *corresponding author: e-mail: gael.cessateur@pmodwrc.ch \\ 2 UJF-Grenoble 1 / CNRS-INSU, Institut de Planétologie et d'Astrophysique de Grenoble (IPAG), UMR 5274, 38041 Grenoble, \\ France \\ 3 LPC2E/CNRS, (UMR 7328) and University of Orléans, 3A avenue de la Recherche Scientifique, 45071 Orléans Cedex 2, France \\ 4 Solar Terrestrial Center of Excellence, Royal Observatory of Belgium (STCE/ROB), Circular 3, 1180 Brussels, Belgium
}

Received 6 March 2012 / Accepted 10 September 2012

\begin{abstract}
Many applications in space weather and in space situational awareness require continuous solar spectral irradiance measurements in the UV, and to a lesser degree in the visible band. Most space-borne solar radiometers are made out of two different parts: (i) a front filter that selects the passband and (ii) a detector that is usually based on silicon technology. Both are prone to degradation, which may be caused either by the degradation of the filter coating due to local deposition or to structural changes, or by the degradation of the silicon detector by solar radiative and energetic particle fluxes. In this study, we provide a theoretical analysis of the filter degradation that is caused by structural changes such as pinholes; contamination-induced degradation will not be considered. We then propose a new instrumental concept, which is expected to overcome, at least partially, these problems. We show how most of the solar UV spectrum can be reconstructed from the measurement of only five spectral bands. This instrumental concept outperforms present spectrometers in terms of degradation. This new concept in addition overcomes the need for silicon-based detectors, which are replaced by wide band gap material detectors. Front filters, which can contribute to in-flight degradation, therefore are not required, except for the extreme-UV (EUV) range. With a small weight and a low telemetry, this concept may also have applications in solar physics, in astrophysics and in planetology.
\end{abstract}

Key words. Space Weather - Solar Spectrum (UV) - Modelling - Instrumentation

\section{Introduction and motivations}

The solar activity manifests itself through several mechanisms. The upcoming cycle may reveal many differences compared to former ones (Podladchikova \& Van der Linden 2011) with many impacts on space weather applications such as GNSS (Sreeja et al. 2011), scintillations (Béniguel \& Hamel 2011) or forecasting (Sigernes et al. 2011; Tsagouri 2011). Among these mechanisms, the solar spectral irradiance in the ultraviolet (UV) is a key quantity for aeronomy throughout the solar system (Lilensten et al. 2008; Mikhailov et al. 2012), especially for evaluating changes in the dayside ionosphere and for forecasting extreme events (Tsurutani et al. 2012). The UV irradiance is also important for the remote sensing of the planets, for climate studies (Fox et al. 2011). In solar physics, the most energetic part of the solar UV spectrum is conventionally divided into middle UV (MUV, 200-300 nm), far UV (FUV, 122$200 \mathrm{~nm}$ ), extreme UV (EUV, 10-121 nm) and soft X-rays (XUV, 0.1-10 nm) (Tobiska \& Nusinov 2006). The solar spectral variability in the UV is highly complex and dynamic, and directly affects the thermosphere/ionosphere system on various time scales. Unfortunately, the long-term monitoring of the UV is a major and expensive challenge: measurements must be carried out in space, where instruments suffer from ageing, degradation and signal contamination. Direct observations are at best sparse, in particular in the EUV range before the launch of the TIMED satellite in 2002. Moreover, the reliability of recent data from SORCE/SIM and SORCE/SOLSTICE is being questioned (e.g., Haigh et al. 2010; Lean et al. 2011).
As of today, all solar UV observations are made either with broadband radiometers or with spectrometers. All these instruments suffer from degradation and are facing the problem of inflight calibration. As a consequence, most applications that require continuous and long-term observations rely instead on a variety of solar proxies that partly mimic some of the spectral bands. The search for more robust instrument concepts therefore is an issue of considerable importance.

The first source of instrument degradation is the contamination of the front filters. All solar missions indeed report an important decrease of the measured irradiance that is most likely caused by the UV polymerisation of hydrocarbon contaminants onto optical surfaces. The EURECA mission, for instance, reported a decrease of about $70 \%$ after 200 days of observation for a filter centred around $330 \mathrm{~nm}$ (Wehrli et al. 1996). The SUSIM spectrometer on board UARS used an $\mathrm{MgF}_{2}$ entrance filter for the $115-263 \mathrm{~nm}$ range. More than $75 \%$ of its signal was lost after 200 days of observation. The instrument scientists eventually completely removed the filter in front of the spectrometer (Floyd, 1999). Recently, similar effects were noticed with the LYRA radiometer on board PROBA2; a major fraction of the signal (of about $90 \%$ ) was lost for the Lyman- $\alpha$ channel after 1 year (Dominique et al. 2012). This degradation is directly related to the cleanliness of the instruments and of the spacecraft itself. Interference filters using quartz or $\mathrm{MgF}_{2}$ material are more prone to such contamination (Floyd 1999). Metallic filters dedicated to EUV range, like zirconium or aluminium, are less affected by it, 
probably because the EUV spectral window is less sensitive to the filter contamination. Degradation studies are notoriously rare in the literature on UV measurements; for that reason, we shall not further address this issue here.

The second main source of instrument degradation is the change in the spectral response of the interference filters, which occurs when their structure changes under the effect of UV radiation or of contamination. As a consequence of this, the spectral information may change in time. Osantowski et al. (1991) characterised the spectral response of several UV filters in the 120-200 nm range, after few months of operation in space. These filters were mode out of using $\mathrm{MgF}_{2}$. In the absence of contamination, they showed that the spectral response decreased by about $30 \%$ around $160 \mathrm{~nm}$, while other wavelengths remained unchanged. Here again, we are suffering from a strong lack of systematic studies.

A third effect is the appearance of pinholes on the surface of metallic filters. Their typical size is in the range of a few micrometers. Such pinholes alter the optical surface, which leads to an increase of the porosity and, by extension, to a modification of the measured signal. Finally, the degradation of silicon detectors is also a function of the absorbed dose of solar energetic radiations and particles, which further reduce instrument life.

To bypass all these problems, several empirical approaches have been developed to reconstruct the solar spectral UV irradiance without direct observations. The most widespread approach is based on the use of solar proxies as substitutes, such as the radio flux at $10.7 \mathrm{~cm}$ (F10.7) (Tapping \& Detracey 1990) or the MgII core-to-wing index (Heath \& Schlesinger 1986), which benefit from long historical records that are relatively well calibrated and are often measured from the ground. Linear combinations of these proxies are used in many empirical models (Hinteregger 1981; Tobiska et al. 2000; Lean et al. 2003; Richards et al. 1994, 2006). Dudok de Wit et al. (2009), however, have shown that no single index, and more importantly, no combination of existing indexes, can properly reconstruct the solar EUV/FUV/MUV irradiance on all time scales, which is quite prejudicial to the characterisation of upper atmospheric parameters (Lilensten et al. 2007). This problem is particularly acute for planetary missions, for which a local measurement of the UV flux (rather than an extrapolation from Earth-based observations) is required to properly characterise local effects.

A second strategy consists in considering the solar spectrums a linear superposition of reference spectra that originate in different regions on the solar disc. Such regions are characterised in terms of structures (such as the quiet Sun, coronal holes or active regions), or, more generally, are based on surface magnetism. They are determined through the analysis of solar images (Warren et al. 1998; Worden et al. 1998; Kretzschmar et al. 2004) or solar magnetograms (Krivova \& Solanki 2008). An operational model (Vieira et al. 2012) ${ }^{1}$, for example, has been developed along that line. As it turns out, only a few types of regions, typically three to four, are sufficient to properly describe the solar spectral variability from the UV to the visible. A statistical analysis has confirmed this unique property (Amblard et al. 2008). On time scales that exceed the dynamic time of transient events such as flares, Floyd et al. (2005) have shown that emissions coming from the upper photosphere, the chromosphere, the transition region and the lower corona are strongly correlated. This coherency stems from the strong

\footnotetext{
${ }^{1}$ Available at the following website: http://lpc2e.cnrs-orleans.fr/ $\sim$ soteria/
}

structuring of the solar atmosphere by the solar magnetic field (Domingo et al. 2009).

A direct consequence of this coherency of the solar spectral variability is the possibility for reconstructing the irradiance at a specific wavelength from nearby wavelengths. The most appropriate set of lines could either be determined using a semi-empirical approach (Kretzschmar et al. 2006) or by a statistical approach (Dudok de Wit et al. 2005). These latter two studies show that a relative error on the reconstructed spectrum as low as $10 \%$ can be achieved with 6-10 lines only. No single instrument, however, measures lines in a strict sense; all have a finite spectral resolution of the order of a few $\mathrm{nm}$ to a few tens of nm. Kretzschmar et al. (2008) first investigated the possibility of using spectral bands (rather than spectral lines) to reconstruct the solar spectral irradiance. Following these studies, Cessateur et al. (2011) extended the concept by testing it with three existing radiometers. It turns out that with four passbands that are properly distributed over the UV spectrum, a relative error below $20 \%$ can be achieved using 6 years of daily-averaged UV spectra from TIMED/SEE (Woods et al. 2005) and SORCE/SOLTICE (Rottman 2005). These studies show that broadband radiometers are excellent candidates for obtaining the solar spectral irradiance in cases where no high spectral resolution is required.

The main issue we shall now focus on is the robustness of broadband detectors regarding degradation. Here, we shall only address the degradation due to solar UV radiation, ignoring the contamination from carbon deposits or other pollutants. There are some technological solutions for overcoming the latter such as cold traps, protecting shields, etc. Our study is organised as follows:

- We first examine the state-of-the-art in the observation of the solar spectrum using radiometers. Two aspects need to be distinguished: detectors and filters. Indeed, both parts are independent and do not suffer a priori from the same kind of degradation.

- We then propose a way to quantify the degradation of the metallic filters by considering their porosity due to the apparition of pinholes. We estimate the degradation considering different technologies.

- We then propose a strategy to overcome these degradations by using detectors without filters.

- We finally propose an instrumental concept for reconstructing the whole UV spectrum.

\section{Technological aspects}

\subsection{Photodetectors}

Space-borne instruments that are dedicated to the observation of the soft X-ray (XUV) to the near-infrared (NIR) spectral bands commonly use Silicon (Si) photodetectors, which are technologically mature. However, present Si photodetectors for the EUV exhibit serious limitations in terms of performance and lifetime.

The recent launch of the ESA technological mission PROBA2 has offered the possibility to test for the first time and in real conditions, photodetectors that are based on wide band gap material (WBGM) technologies (Hochedez et al. 2006). Using WBGM as the active layer makes the photodetectors radiation-hard against both UV radiation and energetic particles (e.g., solar energetic protons, energetic protons and electrons in the radiation belts). These detectors are also partly 
blind to visible light, which alleviates the need for high-rejection filters in the visible range. These are important assets for making observations in the XUV-EUV range.

Photodetectors built out from various WBGM have been reported for a variety of potential applications in the fields of automotive, military defence as well as in environmental and biological research. Among them, SiC (Wright \& Horsfall 2007), GaN (Monroy et al. 1999) and II-VI compound-based detectors (Sou et al. 2001) show a photodetector cut-off wavelength longer than $300 \mathrm{~nm}$, whereas cBN (Soltani et al. 2008), AlN (Butun et al. 2006; Li et al. 2006; Dahal et al. 2007; BenMoussa et al. 2008) and diamond-based (Adam et al. 2004; Balducci et al. 2005; Nesladek 2005; Sio et al. 2005; BenMoussa et al. 2006; Saito et al. 2006; Liao et al. 2007; Keister \& Smedley 2009) devices present a significantly shorter photoresponse cut-off at around 193 (Soltani et al. 2008), 210 (BenMoussa et al. 2008) and $225 \mathrm{~nm}$ (BenMoussa et al. 2006) wavelengths, respectively.

By their nature, $\mathrm{cBN}, \mathrm{AIN}$ and diamond semiconductors are the primary choice as the photosensitive materials for solar EUV and VUV photon detection (spectral range of interest $10 \leq \lambda \leq 200 \mathrm{~nm}$ ) and meet the requirements for UV radiometers planned to study the Sun since they provide high radiation hardness, near-ultraviolet (NUV) and visible-blindness (wavelength cut-off value $\leq 225 \mathrm{~nm}$ ), mechanical, chemical and thermal stability and no need of cooling system (i.e., roomtemperature operation).

Recently, and for upcoming EUV-UV solar radiometers, high-quality $\mathrm{cBN}$, AlN and diamond Metal-SemiconductorMetal (MSM) photodetectors have been built up by the research group of the Royal Observatory of Belgium (BenMoussa et al. 2009a). The aim of this group is to develop innovative photodetectors using the widest band gap materials ${ }^{2}$ that are suited for high temperatures and harsh environments.

There are several important criteria for the design of UV photodetectors for space applications, including the optical wavelength of interest (here $1-280 \mathrm{~nm}$ ), semiconductor material composition and thickness, detector geometrical structure (device architecture) and electrode metal composition. For the purpose of UV radiation detection, it should be noted that the penetration depth $(1 / \alpha(\lambda))$ of almost all semiconductor materials drops drastically to extremely small values, which are of the order of few nanometers. Most photons therefore are absorbed in the first atomic layers, which leads to carrier recombination in the upper surface layer and consequently to a reduction of the sensitivity. Hence, it is crucial to optimise the photo-electron collection close to the surface with a planar configuration of the electrodes. Among different design structures, the MSM represents a simple, cost-effective photodetector design that is not only related to performance but also to the production process. MSM photodetectors are built with coplanar structures, making their production simpler than $p-i-n$ or Schottky diodes that consist of different layers with various doping constituents and concentrations.

\subsection{UV filters}

There are different kinds of filters depending on the spectral ranges. Metallic filters are often used in the EUV range like those based on aluminium and zirconium from the LYRA instrument on board PROBA2. Different architectures can also

\footnotetext{
${ }^{2}$ See, for example, http://bold.oma.be
}

be considered: a thin layer could directly be applied over the detectors, or a mesh grid can be worked out such as porous filters (Dominique et al. 2009) or by using different layers (Auchère et al. 2011). For the FUV and MUV ranges, interference optical filters like those based on quartz or magnesium fluoride $\left(\mathrm{MgF}_{2}\right)$ are often used. Very little information about the nature of these filters, however, is available since it is often patent-dependent (see, for e.g., http://www.princetoninstruments. $\mathrm{com} /$ ).

\subsection{Quantification of the porosity of the filters}

Here, we shall address a simple approach to quantify the impact of the formation of pinholes on filters, which can contribute to in-flight degradation. What does matter here is to compare the performance from silicon-based and wide band gap materialsbased photodetectors for monitoring the solar UV irradiance. Following the work by Dominique et al. (2009), the transmittance of porous filters can be written as

$$
T_{\text {porous filter }}=P\left[1-J_{0}^{2}\left(\frac{2 \pi r^{2}}{\lambda L}\right)-J_{1}^{2}\left(\frac{2 \pi r^{2}}{\lambda L}\right)\right]
$$

with $P$ the porosity of the filter which is the ratio between the area of the holes and the total surface $\left(P=\frac{S_{\text {hole }}}{A_{\text {total }}}\right)$ where by definition $0 \leq P \leq 1 . J_{0}$ and $J_{0}$ are the Bessel functions of the first and second order, $r$ and $L$ are respectively the radius and the average depth of holes. As a first approximation, diffraction effects can be neglected if the hole diameter is larger than the considered wavelengths $\left(2 \pi r^{2} \gg \lambda L\right)$. Mathematically, this implies that these two Bessel functions tend to zero, so that the transmittance for the porous part of the filter can be summarised in one term of porosity $P$. The rest of the filter, non-porous, retains the inherent transmittance of the original material, that is, $T_{\text {material }}$. The transfer function, $F$, the system filter and detector, $D$, can be written as

$$
F=\left[P+(1-P) T_{\text {material }}\right] \times D
$$

We then use a radiometric model (BenMoussa et al. 2009b) based on the solar spectral irradiance measured by TIMED/ SEE, SORCE/SOLSTICE and SIM/SOLSTICE to assess the spectral transmittance of such porous filters associated either with diamond or silicon detectors. Figure 1 displays the total response (filter + detector) for different porosity levels of an aluminium filter. The solar spectrum is also displayed in Figure 1 in order to show the ratio of about 4-5 orders of magnitude between the irradiance as seen in the visible compared to the one observed in the EUV. For relatively high levels (e.g., $P=0.01$ ), the contribution of the visible is nearly of the same order of magnitude for both cases than the nominal spectral band (between 17 and $80 \mathrm{~nm}$ ).

From this, we can also define the purity of a signal as the ratio between the one obtained with the non-porous filter to that obtained with a porous one. As summarised in Table 1, the purity decreases drastically for silicon detectors according to the porosity levels. For porosity levels of about $P=10^{-4}$, the signal variability is similar to that of visible radiation in the case of silicon, while nearly $89 \%$ of the signal can always be attributed to the UV spectral range in the case of diamond detectors. However, for higher porosity, the contribution of the UV signal is drowned in that of the visible regardless of the nature of the 

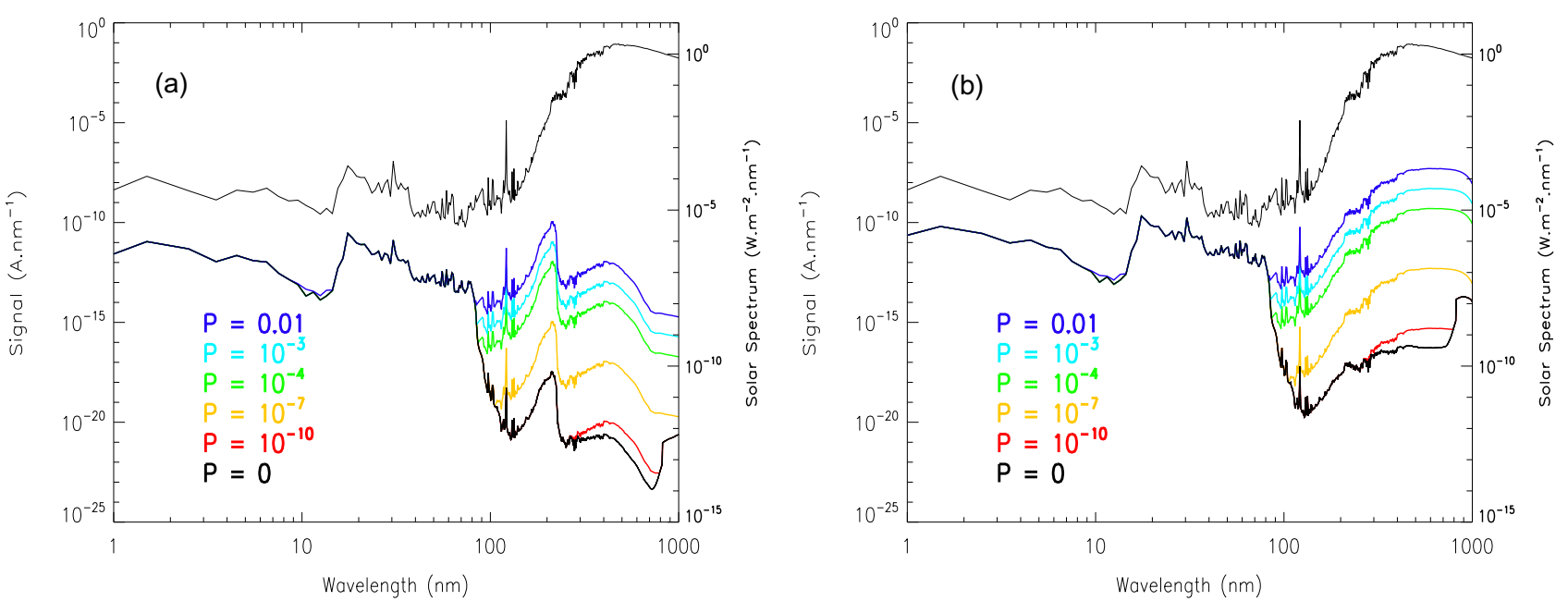

Fig. 1. Spectral response of the system filter-detector (aluminium filter) for several levels of porosity for (a) a diamond detector and (b) a silicon detector for a non-eruptive Sun. The solar spectrum is shown for comparison in black.

Table 1. Purity (\%) obtained for several LYRA channels (their nominal ranges are indicated between parentheses) using either a diamond detector or a silicon detector.

\begin{tabular}{|c|c|c|c|c|c|c|}
\hline \multirow[b]{3}{*}{ Porosity } & \multicolumn{6}{|c|}{ Purity (\%) } \\
\hline & \multicolumn{2}{|c|}{ Aluminium $(17-80 \mathrm{~nm})$} & \multicolumn{2}{|c|}{ Zirconium $(6-20 \mathrm{~nm})$} & \multicolumn{2}{|c|}{ Lyman $\alpha(120-123 \mathrm{~nm})$} \\
\hline & Diamond & Silicon & Diamond & Silicon & Diamond & Silicon \\
\hline 0 & 100 & 100 & 100 & 100 & 100 & 100 \\
\hline $10^{-10}$ & 99.9 & 99.9 & 100 & 100 & 100 & 100 \\
\hline $10^{-7}$ & 99.8 & 86.7 & 99.9 & 99.9 & 99.9 & 9 \\
\hline $10^{-4}$ & 88.9 & 0.63 & 73 & 9.7 & 7.8 & $9 \times 10^{-3}$ \\
\hline $10^{-3}$ & 44.6 & $6 \times 10^{-2}$ & 21 & 1 & 0.8 & $9 \times 10^{-4}$ \\
\hline $10^{-2}$ & 7.5 & $6 \times 10^{-3}$ & 2.5 & $9 \times 10^{-2}$ & $8 \times 10^{-2}$ & $9 \times 10^{-5}$ \\
\hline
\end{tabular}

detector. Similar conclusions can also been made for other passbands of the LYRA instrument (the zirconium and Lyman- $\alpha$ channels). The LYRA instrument uses only diamond PIN detectors for the channel Herzberg (with a nominal range of about $200-220 \mathrm{~nm})$. In this case, the purity is always close to $100 \%$ even for a porosity of about $P=10^{-2}$. This quick study shows the relative importance of the detector for rejecting efficiently the visible range independently from degradation.

In addition to the absolute level of the signal, the information that we seek here is the origin of the solar variability. The lack of purity makes however the attribution of this variability very delicate. As the variability of the solar spectrum is wavelength-dependent, the previous study provides no evidence about the nature of the solar variability as measured by a degraded channel. This severely limits the use of such degraded bandwidth in the reconstruction of the variability of solar irradiance in the UV. A better method is clearly needed to assess the origin of the variability.

We first need to determine which wavelengths behave in a similar way. The analysis method we advocate is identical to the one we used in previous work (Dudok de Wit et al. 2005; Cessateur et al. 2011). Two wavelengths are considered as redundant if the solar irradiance $I(\lambda, t)$ at these two wavelengths, once properly normalised, exhibits the same time-evolution. To this aim, we use a classical technique in multivariate statistics called multidimensional scaling (Chatfield \& Collins 1990), which allows to represent graphically the level of dissimilarity between correlated variables. We first quantify this dissimilarity by a distance. The latter can be defined in different ways; the most familiar measure is Euclidean distance, such that

$$
\delta(i, j)=\sqrt{\sum_{t}\left(\phi\left(\lambda_{i}, t\right)-\phi\left(\lambda_{j}, t\right)\right)^{2}}
$$

where $\phi(\lambda, t)=\frac{I(\lambda, t)-\langle I(\lambda, t)\rangle_{t}}{\sigma_{I_{2}}}$ represents the standardised irradiance at wavelength $\lambda_{I_{\lambda}}{ }^{\prime}\langle\bullet\rangle_{\mathrm{t}}$ denotes time averaging and $\sigma_{I \lambda}$ is the standard deviation of the considered irradiance. When the data are highly correlated, as is the case here, they can be reasonably well represented on a 2-D map in which their pairwise distance reflects their dissimilarity. The smaller the distance is, the more correlated the irradiance at these two wavelengths is and the more likely their common physical origin is. The idea thus is to project our high-dimensional cloud of wavelengths on a 2-D subspace that captures their salient properties in terms of distances.

The location of all the points on the 2-D map can be computed by Singular Value Decomposition (SVD; Golub \& Van Loan 2000). This technique decomposes the normalised irradiances into sets of separable functions of time and wavelength

$$
\phi(\lambda, t)=\sum_{i} W_{i} f_{i}(\lambda) g_{i}(t)
$$

with the condition that these functions are orthonormal, that is,

$$
\left\langle f_{i}(\lambda) f_{j}(\lambda)\right\rangle_{\lambda}=\left\langle g_{i}(t) g_{j}(t)\right\rangle_{t}=\left\{\begin{array}{lll}
1 & \text { if } & i=j \\
0 & \text { if } & i \neq j
\end{array}\right.
$$




\section{G. Cessateur et al.: New observation strategies for the solar UV spectral irradiance}

Each squared weight $W_{i}^{2}$ represents the amount of variance that is described by the $i$ th dimension. Here, the first weights carry a major fraction of the variance thanks to the remarkable redundancy of solar spectral variability. This justifies a priori the projection of all wavelengths on a low-dimensional subspace; the coordinates of each wavelength along the $i$ th dimension (axis) are simply given by $W_{i} f_{i}(\lambda)$. The major advantage of this approach is the possibility to evaluate the dissimilarity between all wavelengths and passbands in a single glance. We shall perform this SVD method to the spectral range of interest, which ranges from 1 to $1130 \mathrm{~nm}$.

Figure 2 shows the 2-D dissimilarity map for time scales below 81 days, which mostly reflect the variability associated with solar rotation. Figure 2a shows the location of the passbands from the LYRA instrument based on silicon, with different levels of porosity. The units are not important here, but the axes do have a physical meaning: the first axis mostly captures the darkening effect of sunspots, while the second axis mostly describes the
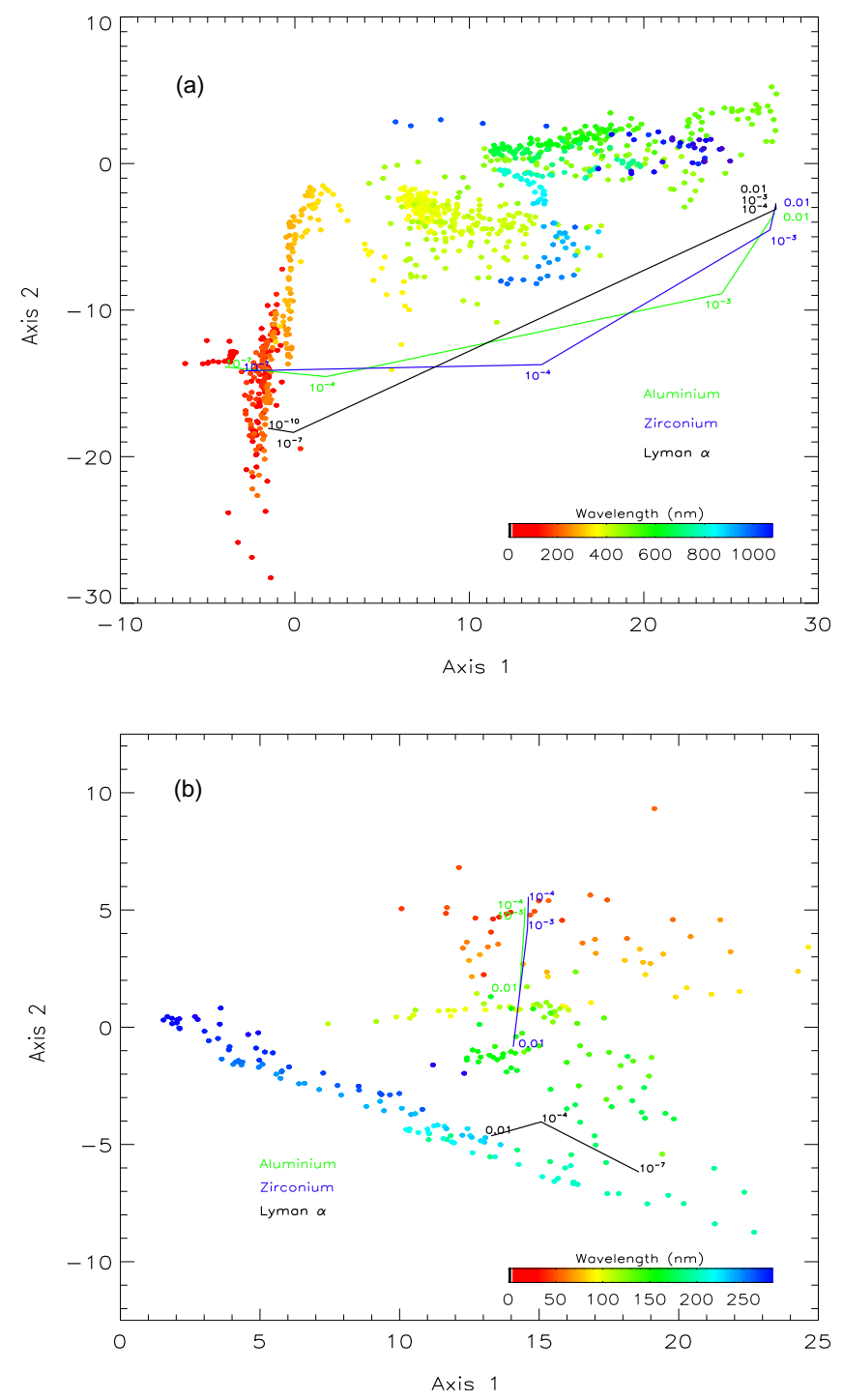

Fig. 2. 2-D representation of the variability of the solar irradiance (a) between 1 and $1000 \mathrm{~nm}$, and (b) between 1 and $280 \mathrm{~nm}$ only. Various channels from the LYRA instrument are overplotted according to, the type of detector: silicon in (a) and diamond in (b). Each channel comes in several porosity levels, which are indicated next to the position of the channel. contribution of bright faculae. Adding more dimensions does not give deeper insight and merely complicates the visualization. What matters is the relative distance between the points and then how the full solar spectrum is organised. Different spectral regions can been identified, expressed by clusters of points that describe specific types of evolution in the solar irradiance. This powerful tool can now be used to evaluate the capacity of detectors with degraded filters to capture the spectral variability. For clarity, the first level of porosity that is suitable for a given passband corresponds to the last level for which no change in the measured variability is observed. For example, if we consider the aluminium channel, all lower levels of porosity below the first one $\left(10^{-7}\right)$ are located at the same position and thus will capture the same variability. Note that for a porosity in excess of $10^{-4}$ $\left(10^{-7}\right.$ for the Lyman- $\alpha$ channel), the variability of the signal substantially deviates from the passband it is supposed to represent. For high levels of porosity, all passbands tend to be dominated by VIS and NIR emissions and thus are no longer of interest for measuring the UV.

Figure $2 \mathrm{~b}$ is similar to Figure $2 \mathrm{a}$, but is now based on diamond detectors and restricted to the $1-280 \mathrm{~nm}$ range. The physical meaning of the axes is also different. Spectral analysis reveals that the first axis approximately describes the 27-day modulation amplitude while the second axis describes the amplitude of the 13.5-day modulation. The latter is partly caused by centre-to-limb variations, which are indeed known to be wavelength-dependent, with, for example, limb darkening in chromospheric lines (Donnelly \& Puga 1990; Crane et al. 2004). Here, as in Figure 2a, we have added the location of degraded passbands. Note that the passband of Lyman $\alpha$ has the highest dispersion among those using diamond detectors. For low levels of porosity, the bandwidth is more sensitive in the MUV. Compared to the previous plot, much higher levels of porosity (typically $10^{-2}$ ) are now needed to observe substantial deviations from ideal case. A relatively larger deviation is observed in the EUV, whose reconstruction is therefore more easily compromised by the impact of porosity. Finally, the Herzberg channel is the most stable, since we observe no significant change in the nature of variability at any level of porosity. Moreover, from a technological point of view, pinholes do not appear in $\mathrm{MgF}_{2}$ substrates.

Figure 2 confirms one of the main advantages of diamond detectors over silicon-based detectors, namely their smaller susceptibility to pinhole degradation of the metallic filter. In particular, for the UV band and for typical levels of porosity, the variability of the signal can be more easily ascribed to that of specific spectral bands. For the case where porous filters are used instead of filters with conventional metal coating, our study shows that wide band gap detectors are to be preferred. Porous filters can indeed be used as low-pass filters, as their cut-off frequency is directly related to the size of the holes.

Our study in only theoretical and too few investigations on the contamination of the optical surface of the filters have been carried out so far. We show, however, that many precautions need to be taken before radiometer data can be used for solar UV spectrum reconstruction. Robustness is a critical issue here, especially for space weather applications. To mitigate the problems of detector degradation, diamond technology offers an interesting alternative to filters such as those using an $\mathrm{MgF}_{2}$ coating, which are less reliable.

Finally, the statistical approach we have presented here offers an interesting tool for evaluating the instrument performance with different filters or detectors. 
Table 2. List of the passbands, with their respective structure and their spectral range in this study.

\begin{tabular}{lrc}
\hline \hline Letter Code & Channel structure & Spectral range $(\mathrm{nm})$ \\
\hline $\mathrm{Al}$ & $\mathrm{Al}_{2} \mathrm{O}_{3} / \mathrm{Al}-$ Diamond & $17-80$ \\
cBN MSM & $\mathrm{cBN}-\mathrm{MSM}$ architecture & $1-193$ \\
cBN PIN & $\mathrm{cBN}-\mathrm{PIN}$ architecture & $130-193$ \\
$\mathrm{AlGaN} \mathrm{1}$ & $\mathrm{AlN} / \mathrm{Al}_{\mathrm{x}} \mathrm{Ga}_{1-\mathrm{x}} \mathrm{N}$ & $210-230$ \\
$\mathrm{AlGaN} \mathrm{2}$ & $\mathrm{Al}_{\mathrm{x}}, \mathrm{Ga}_{1-\mathrm{x}}, \mathrm{N} / \mathrm{Al}_{\mathrm{y}}, \mathrm{Ga}_{1-\mathrm{y}} \mathrm{N}$ & $250-280$ \\
\hline
\end{tabular}

\section{Which instrument for the future?}

The motivation of our study was to investigate new ideas for future radiometers for solar UV monitoring. Future studies will help narrow down their technical constraints. Meanwhile, the remarkable properties of wide band gap materials lead us to propose a new instrumental concept that relies on filterless detectors. Using different detector architectures and different materials, we are able to select theoretically spectral ranges that are adequate for reconstructing the salient features of the solar spectral irradiance in the UV. Table 2 summarises the detectors and their respective spectral ranges we consider in the following. It is important to note that the definition of such spectral ranges is based on the intrinsic properties of the wide band gap materials only. We consider four detectors without front filter for the FUV and MUV ranges; two detectors using cBN (with a cut-off wavelength around $193 \mathrm{~nm}$ ) are indeed dedicated to the FUV range. Two different architectures are available, MSM or PIN, which select the different spectral bands, as explained by BenMoussa et al. (2006).

For the MUV range, we consider a new detector architecture that selects the required passbands by using different materials: aluminium nitride $(\mathrm{AlN})$ and gallium nitride $(\mathrm{GaN})$ with a different stoichiometry (Saito et al. 2009). Because of the particular architecture, no additional windows on the detectors need to be considered for limiting the short wavelength contribution. For the 210-230 nm range, we could also use a diamond detector with a PIN architecture. Wide band gap materials technologies, such as boron nitride (BN), are not mature yet and several issues such as the doping process are still being investigated. To infer the variability of the EUV range, however, front filters are needed because no semiconductors have their gap energy in the EUV range. We consider here aluminium filters, which have already been used for the LYRA instrument (150 nm thick). The use of wide-band gap materials instead of Si allows us to reduce the thickness of such metallic filters.

In order to infer the nature of the variability for such detectors compared to the whole UV range, we use the 2-D map as displayed by Figure 3. What matters here is the relative distance between the points, which represent the spectral irradiance at different wavelengths. The position of the five passbands is also indicated. Detectors made out of wide band gap materials are well distributed over the whole UV spectrum, which is desirable. However, there is little information on wavelengths between 50 and $70 \mathrm{~nm}$, which are located more remotely. This is not surprising as no detector is naturally suited for this particular spectral area. We would probably need one more detector to properly capture the variability in that particular range.

Figure 4 illustrates the reconstruction of the solar spectral irradiance for seven wavelength bins, based on the five passbands from Table 2. The reconstruction method is detailed in Cessateur et al. (2011). The four wavelengths in the EUV range have been chosen because of their importance for terrestrial

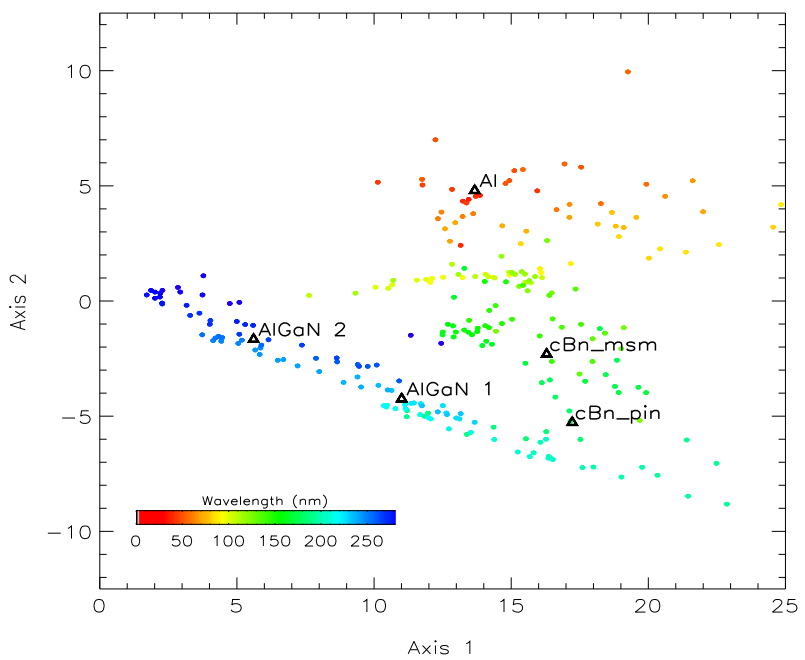

Fig. 3. 2-D representation of the solar variability between 1 and $280 \mathrm{~nm}$ for short time scales. Colour codes correspond to wavelengths $(\mathrm{nm})$. Passbands are indicated by a triangle and by letters (see Table 2).

aeronomy and for solar physics. Lilensten et al. (2007) indeed show which spectral lines are the most important for specifying upper atmospheric parameters such as the neutral density in the thermosphere. These same spectral lines also matter for the reconstruction of the UV irradiance (Dudok de Wit et al. 2005). The irradiance in the $30.5 \mathrm{~nm}$ bin is dominated by the He II line at $30.34 \mathrm{~nm}$ (more than $80 \%$ ) with a significant contribution from coronal lines. The irradiance in the $58.5 \mathrm{~nm}$ is dominated by the $\mathrm{He}$ I line at $58.43 \mathrm{~nm}$. Irradiances in the $97.5 \mathrm{~nm}$ and $102.5 \mathrm{~nm}$ bins are dominated respectively by the optically thick C III line at $97.7 \mathrm{~nm}$ and Lyman- $\beta$ line at $102.57 \mathrm{~nm}$. The $121.5 \mathrm{~nm}$ bin represents the strong Lyman- $\alpha$ line. Finally, the last two irradiance bins represent the contribution of the Si II at $181.69 \mathrm{~nm}$ and the $\mathrm{Mg}$ I absorption edge at $251 \mathrm{~nm}$. Note how both long- and short-term modulations are properly reconstructed. The reconstruction error is below the instrumental precision, except for the $58.5 \mathrm{~nm}$ bin, whose long-term variability is not properly reconstructed.

The 27-day and 13.5-day modulations are also properly reconstructed, except for the few hottest coronal lines such as Fe XVI at $33.4 \mathrm{~nm}$, whose variability is not fully captured by the five detectors. Globally, the solar UV spectrum up to $300 \mathrm{~nm}$ is quite satisfactorily reconstructed and is largely within the instrumental precision, except for some wavelengths in the EUV. Let us mention that this reconstruction is as good as its inputs (from TIMED/SEE and SORCE/SOLSTICE) are. Further investigations are needed to confirm such results with future data sets, and in particular with independent instruments, such as Extreme Ultraviolet Variability Experiment (EVE) on board the Solar Dynamics Observatory (SDO) satellite (Woods et al. 2010).

Such an instrument with broadband measurements is particularly appropriate for monitoring day-to-day variations in the solar UV spectral irradiance, which are essential for characterising the ionosphere/thermosphere system. Typical space weather applications are the monitoring of the thermosphere for satellite drag, and the ionosphere for radio communications.

Note, however, that such broadband observations should be used with care for reconstructing flare spectra. The high correlation between different wavelengths indeed breaks down during energetic events, for which non-flaring relations are not applicable anymore. Only the irradiance in the observed spec- 

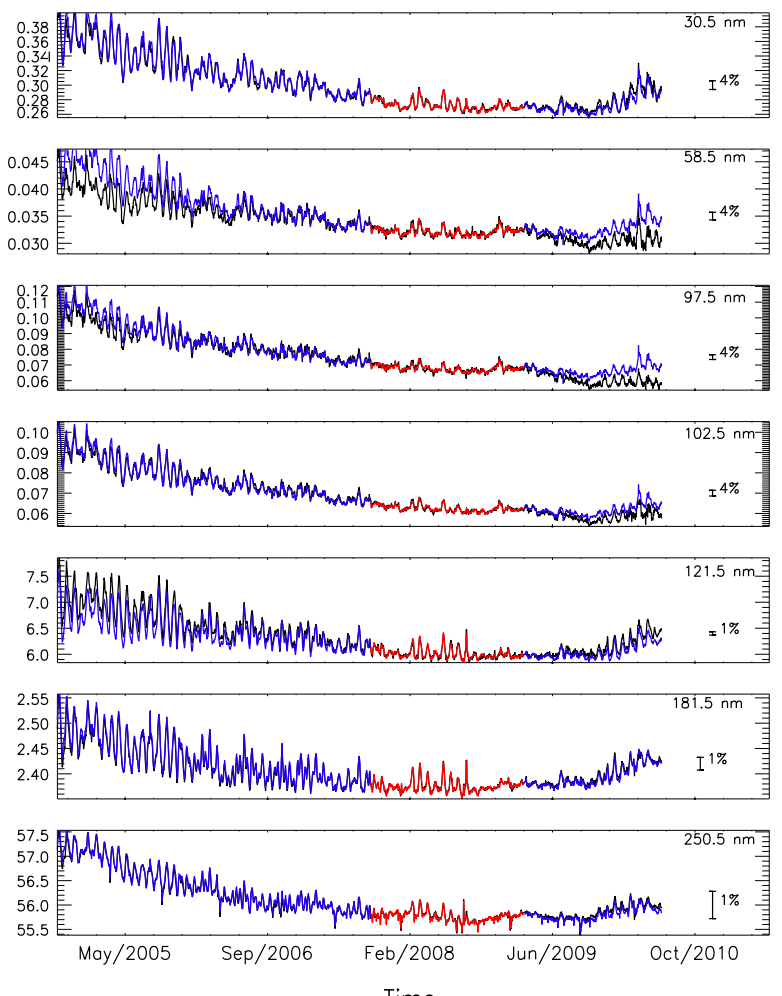

Time

Fig. 4. Comparison between the measured (black) and the reconstructed (colour) spectral irradiance for several wavelength bins that are $1 \mathrm{~nm}$ wide. The coefficients of the linear model are estimated for the one-year interval in red part only. The performance of model really shows up in the blue interval.

tral bands bands will be known exactly. Nevertheless, the instrument output still can be used as a valuable proxy for space weather. The most relevant passband is the $17-80 \mathrm{~nm}$ one, which is already used on LYRA to study flares. For a more precise reconstruction of the flare spectrum one could extend the empirical model by including more inputs, such as soft X-ray channels, as in the Flare Irradiance Spectral Model (FISM; Chamberlin et al. 2008).

Extra care is also required for long-term reconstructions (on time scales of years and beyond) because the correction of detector degradation remains a major challenge, regardless of the detector technology. The usual solutions apply, such as the use of redundant units, cross-calibration between independent instruments, correction with proxy data, etc. Finally, our instrument concept is not adequate for solar spectroscopy, for which high spectral resolution is compulsory.

\section{Conclusion}

Because of the importance of continuous solar spectral irradiance observations in many applications, there remains a considerable issue for developing observation strategies that can overcome the degradation problems that affect present detectors. Here, we show that the use of broadband detectors offers a number of advantages and investigate some of their technological aspects.

Our approach is based on the observational evidence that most of the solar spectral variability can be reconstructed from the measurement of a few spectral bands only. This idea has been developed in several studies (Cessateur 2011 and references herein). Five spectral bands in the UV (as measured by radiometers) are found to be sufficient for retrieving the full UV spectrum with an accuracy that is comparable to that of present spectrometers. Indeed, the selection of these spectral bands has been mostly guided by the physics in order to achieve the smallest error on the reconstructed flux. This observation strategy, however, does not apply to short transients such as flares, for which spectrometers are the best solution.

The selection of these spectral bands here is guided by technological constraints. In this paper, we show which wide band gap materials can properly match the desired spectral bands without relying on front filters. This is a major improvement over traditional instruments, since filters are prone to degradation, with many side effects such as changes in the spectral transmission. Only the EUV range still requires an aluminium filter to properly isolate the desired spectral window. Wide band gap materials, in comparison to standard silicon materials, are also more resilient to structural degradation caused by energetic particles.

The expected advantages of such an instrument are:

- The reduced degradation of the optical system implies a longer lifetime. Such instruments thus are better suited for long-term monitoring of solar activity. In particular, pre-flight calibration should remain valid over a longer time span than for present instruments.

- This new instrument will be much lighter than existing spectrometers. As an example, TIMED/SEE weigh $27 \mathrm{~kg}$ whereas a radiometer with typically four bands and two redundant detectors for each band could weigh within less than $1 \mathrm{~kg}$. For a fair comparison, however, let us stress that their science return is not the same. In particular, spectrometer observations or a spectral model are required to properly calibrate the spectral reconstruction from the radiometer. Other drastic savings are expected for the power budget and for the telemetry requirements. The latter allows for a higher measurement cadence. Given these characteristics, such an instrument could fit as a piggy back option in payloads that are not necessarily Sun-dedicated. Some obvious applications are planetary missions, but also for space weather, for redundant observations are important.

- Finally, such an instrument may find applications in the observation and the reconstruction of the stellar UV spectra. Stellar spectroscopy is nowadays receiving growing attention, one of the reasons being habitability considerations at other planets. An atlas of ground UV spectra of some stars has been recently released (Klochkova et al. 2011).

We are thus advocating the idea that low-weight, low-power, low-cost and small dimensions are not necessarily in contradiction with high reliability. The concept we propose is presently being considered for a small mission that would meet the requirement of the European Space Situational Awareness (SSA) programme. The instrument will be designed for continuous operation whenever the platform is pointing to the Sun, for a regulated DC power bus with a voltage of $28 \mathrm{~V}$ and for a RS422 TM/TC interface with the spacecraft. The instrument will be designed to withstand environmental conditions in near-Earth and interplanetary space (e.g., at L1). The optical design is directly inherited from the PROBA2/LYRA instrument (BenMoussa et al. 2006). The five radiometer channels could 
be mounted in a common structure allowing co-alignment. In each of these channels, the solar flux will be collected through an entrance aperture and a pinhole onto a photodiode detector whose current is converted into a voltage and digitised by an $\mathrm{A} / \mathrm{D}$ converter. For each channel, the resulting signal is proportional to the exposure time and to the solar flux corresponding to the viewing angle in the given passband. All the mechanical parts should be made of $\mathrm{Al}$ alloy as homothetic dilation can be considered in case of thermo-elastic deformations. The field-ofview should be large enough to allow all solar prominences to be covered. Internal calibration sources (LEDs) are used to assess the sensor (visible rejection, UV sensitivity) over time. To ensure stability of the photodiode response and electronics (amplifiers), a controlled heater will be used to stabilise the temperature a few degrees above that of the environment.

This layout could be further detailed but the instrument will be designed to be as flexible and autonomous as possible. It will accommodate (as a piggy back configuration) available resources and environment driven by the primary payload mission and the spacecraft. The total mass, with five channels, should not exceed $1.5 \mathrm{~kg}$, with an objective of $1.0 \mathrm{~kg}$. The preliminary power budget should be less than $1.5 \mathrm{~W}$. These values are estimated from our heritage with similar instruments (in particular LYRA). LYRA weighs $3.53 \mathrm{~kg}$ (total weight per channel is $295 \mathrm{~g}$ ) and measures $315 \mathrm{~mm} \times 92.5 \mathrm{~mm} \times 222 \mathrm{~mm}$. LYRA contains all electronics necessary to control all twelve channels and all three protective covers including the locking mechanism. The average power consumption of LYRA is $3.2 \mathrm{~W}(0.27 \mathrm{~W}$ per channel). Concerning board processing, several concepts developed for LYRA will be probably reused if they make sense, such as storage management, data prioritisation and data compression algorithms.

Our instrumental concept also has several limitations. First of all, it is not suitable for solar spectroscopy. A second limitation is the lack of technological testing, even though the experience gathered from the PROBA2/LYRA and PICARD/PREMOS instruments confirms that the concept works. It is likely, though, that new problems will show up during the research and development phase, and during operation. Let us mention, for example, the problem of the $\mathrm{cBN}$ doping, which needs further investigation. The spectral window of such new detectors, as presented by Saito et al. (2009), is also extremely temperature-dependent. Finally, we are still lacking a complete end-to-end test of their degradation. And, not least, let us mention the ubiquitous problem of hydrocarbon contamination, which has not been addressed here, and presently can be handled only by a drastic control of spacecraft cleanliness. Indeed, the polluted environment of the spacecraft, which alter the filters, may certainly alter the detectors, now unprotected by front filters. Further investigations on this particular issue are a high priority. Redundant observations measurements may partly help overcome some of these problems. We are nevertheless convinced that the present study is an important and necessary step towards the definition of future instruments for SSA programmes.

Acknowledgements. This study received funding from the European Community's Seventh Framework Programme (FP7/2007-2013) under the Grant Agreement No. 218816 (SOTERIA project, www.soteria-space.eu) and grant agreement eHeroes (Project No. 284461, www.eheroes.eu). This study received was also supported by the Swiss National Science Foundation under grant 200020_130102. The authors would like to thank two anonymous referees for comments and suggestions which helped improve this paper.

\section{References}

Adam, W., E. Berdermann, P. Bergonzo, W. de Boer, F. Bogani, et al., New developments in CVD diamond for detector applications, Eur. Phys. J. C-Part. Fields, 33, s1014-s1016, DOI: 10.1140/epjcd/s2004-03-1798-6, 2004.

Amblard, P.-O., S. Moussaoui, T. Dudok de Wit, J. Aboudarham, M. Kretzschmar, J. Lilensten, and F. Auchère, The EUV Sun as the superposition of elementary Suns, $A \& A$, 487, L13-L16, 2008.

Auchère, F., J. Rizzi, A. Philippon, and P. Rochus, Minimization of the shadow patterns produced by periodic mesh grids in extreme ultraviolet telescopes, J. Opt. Soc. Am. A, 28, 40-45, 2011.

Balducci, A., M. Marinelli, E. Milani, M.E. Morgada, A. Tucciarone, G. Verona-Rinati, M. Angelone, and M. Pillon, Extreme ultraviolet single-crystal diamond detectors by chemical vapor deposition, Appl. Phys. Lett., 86 (19), 193509, 2005.

Béniguel, Y., and P. Hamel, A global ionosphere scintillation propagation model for equatorial regions, J. Space Weather Space Clim., 1, A04, 2006.

BenMoussa, A., J.F. Hochedez, U. Schühle, W. Schmutz, K. Haenen, et al., Diamond detectors for LYRA, the solar VUV radiometer on board PROBA2, Diamond Rel. Mater., 15 (48), 802-806, 2006.

BenMoussa, A., J.F. Hochedez, R. Dahal, J. Li, J.Y. Lin, H.X. Jiang, A. Soltani, J.-C. De Jaeger, U. Kroth, and M. Richter, Characterization of AIN metal-semiconductor-metal diodes in the spectral range of 44-360 nm: photoemission assessments, Appl. Phys. Lett., 92 (2), 022108, 2008.

BenMoussa, A., A. Soltani, U. Schühle, K. Haenen, Y.M. Chong, et al., Recent developments of wide-bandgap semiconductor based UV sensors, Diamond Rel. Mater., 18 (5-8), 864, Proceedings of Diamond 2008, the 19th European Conference on Diamond, Diamond-Like Materials, Carbon Nanotubes, Nitrides and Silicon Carbide, 2009a.

BenMoussa, A., I.E. Dammasch, J.-F. Hochedez, U. Schühle, S. Koller, et al., Pre-flight calibration of LYRA, the solar VUV radiometer on board PROBA2, $A \& A$, 508, 1085-1094, $2009 \mathrm{~b}$.

Butun, S., T. Tut, B. Butun, M. Gokkavas, H. Yu, and E. Ozbay, Deep-ultraviolet $\mathrm{Al}_{0.75} \mathrm{Ga}_{0.25} \mathrm{~N}$ photodiodes with low cutoff wavelength, Appl. Phys. Lett., 88 (12), 123503, 2006.

Cessateur, G., Reconstruction du spectre UV solaire en vue de la caractérisation des environnements planétaires, Ph.D. thesis, Université d'Orléans, 2011, October [in French].

Cessateur, G., T. Dudok de Wit, M. Kretzschmar, J. Lilensten, J.-F. Hochedez, and M. Snow, Monitoring the solar UV irradiance spectrum from the observation of a few passbands, $A \& A, \mathbf{5 2 8}$, A68+, 2011.

Chamberlin, P.C., T.N. Woods, and F.G. Eparvier, Flare irradiance spectral model (FISM): Flare component algorithms and results, Space Weather, 6, 5001+, 2008.

Chatfield, C., and A.J. Collins, Introduction to Multivariate Analysis, Chapman and Hall, London, 1990.

Crane, P.C., L.E. Floyd, J.W. Cook, L.C. Herring, E.H. Avrett, and D.K. Prinz, The center-to-limb behavior of solar active regions at ultraviolet wavelengths, $A \& A$, 419, 735-746, 2004.

Dahal, R., T.M. Tahtamouni Al, Z.Y. Fan, J.Y. Lin, and H.X. Jiang, Hybrid AlN-SiC deep ultraviolet Schottky barrier photodetectors, Appl. Phy. Lett., 90 (26), 263505, 2007.

Domingo, V., I. Ermolli, P. Fox, C. Fröhlich, M. Haberreiter, et al., Solar surface magnetism and irradiance on time scales from days to the 11-year cycle, Space Sci. Rev., 145, 337-380, 2009

Dominique, M., A.V. Mitrofanov, J.-F. Hochedez, P. Yu. Apel, U. Schühle, et al., Track membranes with open pores used as diffractive filters for space-based x-ray and EUV solar observations, Appl. Opt., 48 (5), 834-841, 2009.

Dominique, M., J.-F. Hochedez, W. Schmutz, I.E. Dammasch, A.I. Shapiro, M.A. Kretzschmar, D. Benmoussa Gillotay, and Y. Stockman, The LYRA instrument on-board PROBA2: description and in-flight performances, Sol. Phys., Submitted, 2012. 
Donnelly, R.F., and L.C. Puga, Thirteen-day periodicity and the center-to-limb dependence of UV, EUV, and X-ray emission of solar activity, 130, 369-390, 1990.

Dudok de Wit, T., J. Lilensten, J. Aboudarham, P.-O. Amblard, and M. Kretzschmar, Retrieving the solar EUV spectrum from a reduced set of spectral lines, Ann. Geophys., 23, 3055-3069, 2005.

Dudok de Wit, T., M. Kretzschmar, J. Lilensten, and T. Woods, Finding the best proxies for the solar UV irradiance, Geophys. Res. Lett., 36, 10107+, 2009.

Floyd, L., Filter responsivity degradation caused by solar UV exposure, Adv. Space Res., 23, 1459-1462, 1999.

Floyd, L., J. Newmark, J. Cook, L. Herring, and D. McMullin, Solar EUV and UV spectral irradiances and solar indices, J. Atmos. Sol.Terr. Phys., 67, 3-15, 2005.

Fox, N., A. Kaiser-Weiss, W. Schmutz, K. Thome, D. Young, B. Wielicki, R. Winkler, and E. Woolliams, Accurate radiometry from space: an essential tool for climate studies, Phil. Trans. $R$. Soc. A: Math. Phys. Eng. Sci., 369 (1953), 4028-4063, 2011.

Golub, G.H., and C.F. Van Loan, Matrix Computations, Johns Hopkins Press, Baltimore, 2000.

Haigh, J.D., A.R. Winning, R. Toumi, and J.W. Harder, An influence of solar spectral variations on radiative forcing of climate, Nature, 467, 696-699, 2010

Heath, D.F., and B.M. Schlesinger, The Mg 280-nm doublet as a monitor of changes in solar ultraviolet irradiance, J. Geophys. Res., 91, 8672-8682, 1986.

Hinteregger, H.E., Representations of solar EUV fluxes for aeronomical applications, Adv. Space Res., 1, 39-52, 1981.

Hochedez, J.-F., W. Schmutz, Y. Stockman, U. Schühle, A. Benmoussa, et al., LYRA, a solar UV radiometer on Proba2, Adv. Space Res., 37, 303-312, 2006.

Keister, J.W., and J. Smedley, Single crystal diamond photodiode for soft X-ray radiometry, Nucl. Instrum. Methods Phys. Res., A: Accel. Spectrom. Detect. Assoc. Equip., 606 (3), 774-779, 2009.

Klochkova, V.G., E.L. Chentsov, T. Kipper, V.E. Panchuk, N.S. Tavolganskaya, and M.V. Yushkin, An atlas of ground UV spectra of selected stars, Astrophys. Space Sci., 335, 83-89, 2011.

Kretzschmar, M., J. Lilensten, and J. Aboudarham, Variability of the EUV quiet Sun emission and reference spectrum using SUMER, $A \& A, 419,345-356,2004$.

Kretzschmar, M., J. Lilensten, and J. Aboudarham, Retrieving the solar EUV spectral irradiance from the observation of 6 lines, $A d v$. Space Res., 37, 341-346, 2006.

Kretzschmar, M., T. Dudok de Wit, J. Lilensten, J.-F. Hochedez, J. Aboudarham, P.-O. Amblard, F. Auchère, and S. Moussaoui, Solar EUV/FUV irradiance variations: analysis and observational strategy, Acta Geophys., 57, 42-51, 2008.

Krivova, N.A., and S.K. Solanki, Models of solar irradiance variations: current status, J. Astrophys. Astron., 29, 151-158, 2008.

Lean, J.L., H.P. Warren, J.T. Mariska, and J. Bishop, A new model of solar EUV irradiance variability 2. Comparisons with empirical models and observations and implications for space weather, J. Geophys. Res. (Space Phys.), 108, 1059+, 2003.

Lean, J.L., T.N. Woods, F.G. Eparvier, R.R. Meier, D.J. Strickland, J.T. Correira, and J.S. Evans, Solar extreme ultraviolet irradiance: present, past, and future, J. Geophys. Res. (Space Phys.), 116, A01102, 2011.

Li, J., Z.Y. Fan, R. Dahal, M.L Nakarmi, J.Y. Lin, and H.X. Jiang, $200 \mathrm{~nm}$ deep ultraviolet photodetectors based on AlN, Appl. Phys. Lett., 89 (21), 213510, 2006.

Liao, M., Y. Koide, and J. Alvarez, Single Schottky-barrier photodiode with interdigitated-finger geometry: Application to diamond, Appl. Phys. Lett., 90 (12), 123507, 2007.

Lilensten, J., T. Dudok de Wit, P.-O. Amblard, J. Aboudarham, F. Auchère, and $\mathrm{M}$. Kretzschmar, Recommendation for a set of solar EUV lines to be monitored for aeronomy applications, Ann. Geophys., 25 (6), 1299-1310, 2007.
Lilensten, J., T. Dudok de Wit, M. Kretzschmar, P.-O. Amblard, S. Moussaoui, J. Aboudarham, and F. Auchère, Review on the solar spectral variability in the EUV for space weather purposes, Ann. Geophys., 26, 269-279, 2008.

Mikhailov, A.V, A. Belehaki, L. Perrone, B. Zolesi, and I. Tsagouri, Retrieval of thermospheric parameters from routine ionospheric observations: assessment of method's performance at midlatitudes daytime hours, J. Space Weather Space Clim., 2, A03, 2012.

Monroy, E., F. Calle, E. Muoz, F. Omns, B. Beaumont, and P. Gibart, Visible-blindness in photoconductive and photovoltaic $\mathrm{AlGaN}$ ultraviolet detectors, J. Electron. Mater., 28, 240-245, DOI: $10.1007 / \mathrm{s} 11664-999-0021-2,1999$.

Nesladek, M., Conventional $n$-type doping in diamond: state of the art and recent progress, Semicond. Sci. Technol., 20 (2), R19, 2005.

Osantowski, J.F., R.A.M. Keski-Kuha, H. Herzig, A.R. Toft, J.S. Gum, and C.M. Fleetwood, Optical coating technology for the EUV, Adv. Space Res., 11, 185-201, 1991.

Podladchikova, T., and R. Van der Linden, An upper limit prediction of the peak sunspot number for solar cycle 24, J. Space Weather Space Clim., 1, A01, 2011.

Richards, P.G., J.A. Fennelly, and D.G. Torr, EUVAC: a solar EUV flux model for aeronomic calculations, J. Geophys. Res., 99, 8981-8992, 1994.

Richards, P.G., T.N. Woods, and W.K. Peterson, HEUVAC: A new high resolution solar EUV proxy model, Adv. Space Res., 37, 315-322, 2006

Rottman, G., The SORCE mission, Sol. Phys., 230, 7-25, 2005.

Saito, T., K. Hayashi, H. Ishihara, and I. Saito, Characterization of photoconductive diamond detectors as a candidate of FUV/VUV transfer standard detectors, Metrologia, 43 (2), S51, 2006.

Saito, T., T. Hitora, H. Hitora, H. Kawai, I. Saito, and E. Yamaguchi, UV/VUV photodetectors using group III-nitride semiconductors, Phys. Stat. Sol. C, 6 (S2), S658-S661, 2009.

Sigernes, F., M. Dyrland, P. Brekke, S. Chernouss, D.A. Lorentzen, K. Oksavik, and C. Sterling Deehr, Two methods to forecast auroral displays, J. Space Weather Space Clim., 1, A03, 2011.

Sio, A, J. De Achard, J. De Achard, A. Tallaire, R.S. Sussmann, A.T. Collins, F. Silva, and E. Pace, Electro-optical response of a singlecrystal diamond ultraviolet photoconductor in transverse configuration, Appl. Phys. Lett., 86 (21), 213504, 2005.

Soltani, A., H.A. Barkad, M. Mattalah, B. Benbakhti, J.-C. De Jaeger, $193 \mathrm{~nm}$ deep-ultraviolet solar-blind cubic boron nitride based photodetectors, Appl. Phys. Lett., 92 (5), 053501, 2008.

Sou, I.K., C.W. Wu Marcus, T. Sun, K.S. Wong, and G.K.L. Wong, Molecular-beam-epitaxy-grown $\mathrm{ZnMgS}$ ultraviolet photodetectors, Appl. Phys. Lett., 78 (13), 1811-1813, 2001.

Sreeja, V., M. Aquino, B. Forte, Z. Elmas, C. Hancock, et al., Tackling ionospheric scintillation threat to GNSS in Latin America, J. Space Weather Space Clim., 1, A05, 2011.

Tapping, K.F., and B. Detracey, The origin of the 10.7 CM flux, Sol. Phys., 127, 321-332, 1990 .

Tobiska, W., and A. Nusinov, ISO 21348 - process for determining solar irradiances, Pages 2621-+ of: 36th COSPAR Scientific Assembly, 36, COSPAR, Plenary Meeting, 2006.

Tobiska, W.K., T. Woods, F. Eparvier, R. Viereck, L. Floyd, D. Bouwer, G. Rottman, and O.R. White, The SOLAR2000 empirical solar irradiance model and forecast tool, J. Atmos. Sol.-Terr. Phys., 62, 1233-1250, 2000.

Tsagouri, I., Evaluation of the performance of DIAS ionospheric forecasting models, J. Space Weather Space Clim., 1, A02, 2011.

Tsurutani, B.T., O.P. Verkhoglyadova, A.J. Mannucci, G.S. Lakhina, and J.D. Huba, Extreme changes in the dayside ionosphere during a Carrington-type magnetic storm, J. Space Weather Space Clim., 2, A05, 2012. 
Vieira, L.E., T. Dudok de Wit, and M. Kretzschmar, Short-term forecast of the total and spectral solar irradiance, J. Space Weather Space Clim., Submitted, 2012.

Warren, H.P., J.T. Mariska, and J. Lean, A new reference spectrum for the EUV irradiance of the quiet Sun 1. Emission measure formulation, J. Geophys. Res., 103, 12077-12090, 1998.

Wehrli, C., C. Fröhlich, and J. Romero, Space degradation of SOVA sunphotometers on EURECA, Metrologia, 32, 653-656, 1996.

Woods, T.N., F.G. Eparvier, S.M. Bailey, P.C. Chamberlin, J. Lean, G.J. Rottman, S.C. Solomon, W.K. Tobiska, and D.L. Woodraska, Solar EUV Experiment (SEE): Mission overview and first results, J. Geophys. Res. (Space Phys.), 110, 1312+, 2005.
Woods, T.N., F.G. Eparvier, R. Hock, A.R. Jones, D. Woodraska, et al., Extreme ultraviolet variability experiment (EVE) on the solar dynamics observatory (SDO): overview of science objectives, instrument design, data products, and model developments, Sol. Phys., 275 (1-2), 115-143, 2010.

Worden, J.R., O.R. White, and T.N. Woods, Evolution of chromospheric structures derived from $\mathrm{Ca}$ II $\mathrm{K}$ spectroheliograms: implications for solar ultraviolet irradiance variability, Astrophys. J., 496, 998+, 1998.

Wright, N.G., and A.B. Horsfall, SiC sensors: a review, J. Phys. D: Appl. Phys., 40 (20), 6345, 2007. 